\title{
All-inorganic perovskite quantum dot light-emitting memories
}

\section{Ya-Ju Lee ( $\nabla$ yajulee@ntnu.edu.tw )}

Institute of Electro-Optical Engineering/National Taiwan Normal University, https://orcid.org/00000002-2663-682X

\section{Kang-Hsiang Liu}

Institute of Electro-Optical Engineering/National Taiwan Normal University,

\section{Yi Peng}

Institute of Electro-Optical Engineering/National Taiwan Normal University,

\section{Meng-Cheng Yen}

Institute of Electro-Optical Engineering/National Taiwan Normal University,

\section{Junfu Leng}

Institute for Materials Chemistry and Engineering (IMCE), Kyushu University

\section{Chun-Chieh Chang}

Institute of Electro-Optical Engineering, National Taiwan Normal University

\section{Kaoru Tamada}

Kyushu University https://orcid.org/0000-0003-2618-9924

\section{Article}

Keywords: perovskite quantum dots (QDs), optoelectronics

Posted Date: December 31st, 2020

DOl: https://doi.org/10.21203/rs.3.rs-128974/v1

License: (c) (1) This work is licensed under a Creative Commons Attribution 4.0 International License. Read Full License

Version of Record: A version of this preprint was published at Nature Communications on July 22nd, 2021. See the published version at https://doi.org/10.1038/s41467-021-24762-w. 


\title{
All-inorganic perovskite quantum dot light-emitting memories
}

\author{
Ya-Ju Lee ${ }^{1,}{ }^{*}$, Kang-Hsiang Liu ${ }^{1}$, Yi Peng ${ }^{1}$, Meng-Cheng Yen ${ }^{1}$, Junfu Leng ${ }^{2}$, Chun-Chieh Chang ${ }^{1,{ }^{*}}$ \& \\ Kaoru Tamada ${ }^{2, *}$ \\ ${ }^{1}$ Institute of Electro-Optical Engineering, National Taiwan Normal University, Taipei, 11677, Taiwan \\ ${ }^{2}$ Institute for Materials Chemistry and Engineering (IMCE), Kyushu University, 744 Motooka, Nishiku, Fukuoka \\ 819-0395, Japan
}

\begin{abstract}
Field-induced ionic motions in all-inorganic $\mathrm{CsPbBr}_{3}$ perovskite quantum dots (QDs) strongly dictate not only their electro-optical characteristics but also the ultimate device performance of the $\mathrm{CsPbBr}_{3}$ optoelectronics. Novel device concepts with multiple functionalities can as a result be achieved based on $\mathrm{CsPbBr}_{3}$ by carefully controlling the ionic flow under different bias conditions. Here, we show that by manipulating the ion migrations in two nominally identical, series-connected $\mathrm{Ag} / \mathrm{CsPbBr}_{3} / \mathrm{ITO}$ devices, one device can operate as a resistive random-access memory (RRAM) while the other simultaneously as a light-emitting electrochemical cell (LEC), or vice versa, simply through the polarity switching of the external bias across the entire structure. We further show that this electrically switchable, seriesconnected perovskite memories and light emitters can be employed as a novel all-perovskite light-emitting memory (LEM) device for simultaneous electronic and optical reading of the encoded information in communication and computation applications. We present a physical picture that clearly depicts the movements of each ionic species and their reduction or oxidation processes in the perovskite LEM responsible for the observed electronic and optical characteristics. The demonstrated bifunctionality of the simple metal-perovskite-metal structures and the novel device concept derived from their creative synergy opens up a completely new horizon for more advanced all-inorganic perovskite optoelectronics with novel functionalities.
\end{abstract}

\footnotetext{
* Correspondence and requests for materials should be addressed to: yajulee@ntnu.edu.tw, chang48@ntnu.edu.tw, tamada@ms.ifoc.kyushu-u.ac.jp
} 


\section{Introduction}

Recently, the demand for the next-generation memory devices featuring high switching speed, large storage capacity, and low power consumption has considerably grown, as the internet of things (IoT) and artificial intelligence (AI) technologies continue to dominate for a vast variety of consumer products used in day-to-day life. To meet this ever-increasing requirement, various kinds of high-performance advanced memory devices have been eagerly pursued in the semiconductor industry. Among them, the nonvolatile resistive random-access memory (RRAM, in which the stored information does not vanish when the applied power is turned off) constructed by simple metal/insulator/metal thin-film stacks, is an essential building block for this digital revolution evolving complicated algorithms ${ }^{1-3}$. The current-voltage $(I-V)$ characteristics of the RRAM exhibit an abrupt change in electrical resistance between a high-resistance state (HRS) and a low-resistance state (LRS). One possible mechanism causing such a disparity is attributed to the formation and annihilation of conductive filament bridging the two metal electrodes under the variation of the applied electric field (including both direction and magnitude) across the RRAM, which consequently leads to the desirable electric-field-driven resistive switching. The HRS and LRS of the RRAM, as analogous to the basic Boolean true and false expressions, are then used to represent the logic " 0 " and " 1 " signals in the digital electronic circuits, respectively. As the readout process of these two logic signals in a RRAM relies on the resistance measurement in a series sequence triggered by an external bias, the overall transmitted data rate using RRAM is fundamentally limited. A modified RRAM scheme that allows and compiles the parallel reading processes is therefore highly anticipated.

Optically readable memories, which integrate a conventional RRAM with a light-emitting diode (LED), are recently developed to overcome this issue, in which the HRS and LRS of the RRAM are recognized directly by the absence or presence of electroluminescence $(E L)$ emissions from the $\mathrm{LED}^{4-8}$. Such a combination of the RRAM with the LED brings about a new memory device dubbed light-emitting memory (LEM), which enables parallel and synchronous reading processes of encoded signals via both electrical and optical detections. Compared to the RRAM solely using the electronic reading, the higher bandwidth enabled by the additional optical reading of the LEM offers much larger flexibility to carry more information and to realize multilevel data storage ${ }^{9-11}$. Additionally, the noncontact optical read-out and the high-speed optical transmission inherently in the LEM are expected to extend the functionality of the conventional RRAM, especially in the fields of computation and communication ${ }^{12,13}$. However, the previous LEMs proposed in the literature are usually produced by integrating two separate devices comprised of completely dissimilar material systems. Many restrictions and issues arise in such hybrid LEMs in terms of their manufacturing compatibility, fabrication simplicity, and transmission synchronization of electrical and optical signals. Therefore, there is an urgent need for a novel LEM structure based on one specific material 
facilitating seamless integration between the photonic system and the electrical resistive switching for overcoming these challenges.

To date, all-inorganic metal halide perovskite materials, usually expressed as the chemical formula of $\mathrm{ABX}_{3}$, where $\mathrm{A}$ and $\mathrm{B}$ are metal cations with different sizes and $\mathrm{X}$ being a halide anion, have been intensively investigated due to their unique photophysical characteristics and high stability in the ambient environment ${ }^{14,15}$. They can be easily processed by solution methods and be implemented in low-cost, mass-production of optoelectronic devices for high-scalable applications. Furthermore, all-inorganic perovskite halides have low activation energies for the migration of halide-ion vacancies under an applied bias, which enables the drifts of charged cations and anions per the poling direction and consequently, forms a reversible p-i-n homojunction structure ${ }^{16,17}$. Although a unified interpretation of the activation energy and the identification of actual moving species remains elusive, these intriguing field-induced ionic motions are believed to be the cause of several unusual phenomena observed in perovskite optoelectronic devices, for example, the hysteresis in $I$ - $V$ curves observed in perovskite solar cells ${ }^{18,19}$ and the ionization-doping enhanced $E L$ emission in the perovskite light-emitting electrochemical cell (LEC) ${ }^{20-22}$. There are also plenty of reports showing that the high ionic conductivity associated with the migration of halide-ion vacancies can lead to significant resistive switching in the perovskite RRAM ${ }^{23-25}$. To further elucidate the underlying mechanism behind the complex ionic motions of various species in perovskites and to advance the fledgling LEM technologies to the next level, here we propose and demonstrate a novel all-inorganic perovskite $\mathrm{CsPbr}_{3} \mathrm{LEM}$ structure by monolithically integrating a perovskite LEC with a perovskite RRAM. We show that this all-perovskite LEM device could potentially be employed for simultaneous optical and electrical reading of encoded information, thereby enabling much faster data processing and much larger storage space. We further present a physical picture outlining the movements of each ion in the perovskite LEM structure and their reduction or oxidation processes under different bias scenarios by carefully investigating its intriguing electronic and optical characteristics. As the functionality of each perovskite device in the LEM structure is switchable (i.e., LEC switches to RRAM, or vice versa) depending on the field direction across the LEM, the proposed device scheme shows extraordinary modulation ability that could lead to new applications for the all-inorganic metal halide perovskite materials.

\section{Results and Discussion}

Figure 1a schematically presents the configuration of our all-inorganic perovskites-based LEM (top plot), composed of two nominally identical devices with a dimension of $\sim 3.0 \mathrm{~mm} \times 3.0 \mathrm{~mm}$. Each has dual functionalities either as RRAM or LEC, completely switchable by manipulating the electric field direction across the LEM (bottom plot, Fig. 1a), as can be seen later in the article. A set of two devices is fabricated on top of a glass substrate with 250-nm-thick indium tin oxide (ITO) rectangular 
pads $(4.0 \mathrm{~mm} \times 7.0 \mathrm{~mm})$, formed by RF magnetron sputtering through a shadow mask. Although the spatial distance between two devices is $\sim 1.0 \mathrm{~mm}$, they are connected electrically in series as the ITO has a low resistivity of $\sim 4.8 \times 10^{-4} \Omega \cdot \mathrm{cm}$. Then, close-packed $\mathrm{CsPbBr}_{3}$ QDs synthesized by the supersaturated recrystallization (SR) method at room temperature (see Methods) are spun coated on top of the ITO pads at $3000 \mathrm{rpm}$ for 30 seconds, forming a quasi-continuous film with a thickness of $\sim 800$ $\mathrm{nm}$ for the LEM device active layer. Here the $\mathrm{CsPbBr}_{3} \mathrm{QDs}$ are preferred than bulk perovskites since, in order to form the latter, the perovskite precursor solution would need to be dispersed directly on the sample followed by a post-annealing process for facilitating the nucleation and crystallization of the bulk perovskites ${ }^{26-28}$, but the heating process would introduce undesirable thermal stress to the underlying ITO and reduce the electrical conductivity of ITO ${ }^{29,30}$ and degrade the overall LEM device performance. Additionally, the perovskites QDs would offer a great opportunity compared to its bulk counterpart for probing the inherent quantum confinement effect on the device's performance. A polymethyl methacrylate (PMMA) protection layer is then coated on top of the $\mathrm{CsPbBr}_{3}$ QDs at the same spin speed. Finally, a 100-nm-thick circle-patterned ( 1.0 mm in diameter) silver (Ag) electrode, usually implemented for increasing the ON/OFF ratio of the RRAM ${ }^{31}$, is deposited on top of the PMMA as contact electrodes by RF magnetron sputtering. Sharp boundaries between $\mathrm{ITO} / \mathrm{CsPbBr} \mathrm{QD}_{3} \mathrm{QD}$ and $\mathrm{PMMA} / \mathrm{CsPbBr} \mathrm{Q}_{3} \mathrm{QDs}$ layers are well distinguished and clearly observed (Fig. 1b). The X-ray diffraction (XRD) pattern of the $\mathrm{CsPbBr}_{3} \mathrm{QDs}_{\mathrm{P}}$ exhibits a monoclinic crystal phase, which is similar to that of the cubic structure (JSPDS No. 54-0752) and can be discriminated by the split of the diffraction peak at $\approx 31^{\circ}$ corresponding to $(200)$ plane reflection, as shown in Fig. 1c. This indicates the good crystallographic quality and successful synthesis of the $\mathrm{CsPbBr}_{3}$ QDs. The crystalline structure and the arrangement of the atomic plane of the $\mathrm{CsPbBr}_{3}$ QDs are further examined by using transmission electron macroscope (TEM). The as-synthesized $\mathrm{CsPbBr}_{3}$ QDs tend to aggregate with each other (Fig. 1d), and clear fused interface can be observed between two adjacent QDs (Fig. 1e). These results suggest the transfer of $\mathrm{Cs}^{+}, \mathrm{Pb}^{2+}$, and $\mathrm{Br}^{-}$ions from the soluble to insoluble solvents is too fast during the supersaturated recrystallization process, which significantly enlarges the QD size to $\sim 50-100 \mathrm{~nm}$, larger than the $\mathrm{CsPbBr} 3$ QDs synthesized by the hot-injection method that generally have a smaller size of $\sim 10-15 \mathrm{~nm}^{14,15}$. Nevertheless, the wellresolved lattice fringes with an average interplanar space of $0.59 \mathrm{~nm}$ can be observed (Fig. 1e), consistent with the distance between the (200) planes indexed in the XRD measurement. Notably, the dimensions of $\mathrm{CsPbBr}_{3}$ QDs with high crystallization are similar to the grain size of the perovskite films produced by the precursor solution with post-annealing treatment ${ }^{26-28}$, suggesting that the $\mathrm{CsPBr}_{3}$ QDs are suitable not only for the LEM device active layer, but also for many other perovskite optoelectronic devices ${ }^{32,33}$. The elemental composition of the $\mathrm{CsPbBr}_{3}$ QDs is also analyzed by using the energy dispersive $\mathrm{X}$ ray spectroscopy (EDS), as shown in Fig. 1g. The EDS mapping clearly illustrates that the $\mathrm{Cs}$, $\mathrm{Pb}$, and $\mathrm{Br}$ are homogeneously distributed inside the $\mathrm{CsPBBr}_{3}$ QDs with the ratio of elemental amount as $20(\mathrm{Cs}): 15(\mathrm{~Pb}): 65(\mathrm{Br})$. A sharp emission peak 
centered at $\lambda=526 \mathrm{~nm}$ with a comparable photoluminescence (PL) intensity is observed in both the $\mathrm{CsPbBr} \mathrm{QDS}_{3}$ and PMMA/CsPbBr 3 QDs samples (Fig. 1f). A photograph of the fabricated LEM devices taken under the illumination of 405 nm UV-LED is also inserted in the figure. The same amount of Stokes shift of $\sim 8 \mathrm{~nm}$ is therefore determined for both $\mathrm{CsPbBr}_{3}$ QDs samples with and without protective PMMA as they have a similar excitonic absorption peak located at $\lambda=518 \mathrm{~nm}$. The PMMA protection layer in the structure provides an effective way to prevent the Ag penetration during the sputtering deposition of Ag electrode, as evidenced by Fig. S1, which in turn minimizes the formation of current leakage paths between the top (Ag) and the bottom (ITO) electrodes, and enhances the performance and the stability of the fabricated LEM. The surface morphologies of the $\mathrm{CsPbr}_{3}$ QDs, the PMMA /CsPbBr 3 QDs, and the $\mathrm{Ag} / \mathrm{PMMA} / \mathrm{CsPbBr}_{3}$ QDs films are also examined by the atomic force microscope (Fig. S2, Supplementary Information).

We first investigate the emission characteristics of the LEM by applying a negative poling voltage on one of its constituent devices through the top Ag (cathode) and the bottom ITO (anode) electrodes. Since the perovskite materials are conceived as a class of solid electrolytes with good ionic conductivity, electric dipoles pointing along the direction of the electric field (bottom-up) can be induced inside the active layer, as plotted in Fig. 2a. Besides, the cations (i.e., $\mathrm{Cs}^{+}$or bromide ion vacancy, $\mathrm{V}_{\mathrm{Br}}{ }^{+}$) and anions (i.e., $\mathrm{Br}^{-}$) are further dissociated from the $\mathrm{CsPbBr}_{3}$ QDs and which would drift, respectively, towards the Ag cathode and the ITO anode and accumulate there. The band bending of the $\mathrm{CsPbBr}_{3} \mathrm{QDs}$ at the interfaces is hence changed accordingly and leads to the formation of p-i-n diode (inset, Fig. 2b), which facilitates the photon emission through the radiative recombination of electron-hole pairs, and that is the working principle of the $\mathrm{CsPbBr}_{3}$ QD-based LEC. Here shall be addressed that light emission can only be observed by applying the negative poling voltage on the LEC, while no light is emitted if changing the voltage polarity to positive. Figure $2 \mathrm{~b}$ shows the typical $I$ - $V$ characteristics of the LEC under the negative poling voltage sweeping from 0 to $8 \mathrm{~V}$ (black curve, forward $I-V$ ) and then sweeping back from 8 to $0 \mathrm{~V}$ (red curve, reverse $I-V$ ). Both forward and reverse $I$ - $V$ characteristics exhibit well rectifying diode-like behaviors, suggesting a proper formation of p-i-n homojunctions ${ }^{34}$. Also, a clear electrical hysteresis effect is observed in this figure, mainly because the cations and anions accumulated at the cathode and anode cannot respond instantaneously to the applied voltage change. Note that no pre-poling voltage is applied to the LEC before acquiring its $I$ - $V$ characteristic (Fig. 2b), and the hysteresis effect is observed upon biasing the LEC and is repeatable after multiple sweepings. In Fig. 2c, we replot $I-V$ characteristics of the LEC in a semi-log scale to identify several physical parameters by the modified Shockley equation expressed as ${ }^{35}$ :

$$
I-\left[\left(V-I R_{S}\right) / R_{P}\right]=I_{S} \exp \left[q\left(V-I R_{S}\right) / n_{\text {ideal }} k T\right]
$$


where $R_{S}, R_{P}, n_{\text {ideal }}$, and $I_{S}$ are the series resistance, shunt resistance, ideality factor, and saturation current, respectively. The turn-on voltage, series and shunt resistances extracted from the forward and reverse $I$ - $V$ characteristics are $V_{\text {turn-on }}=3.8 \mathrm{~V}$, $R_{S}=104.7 \Omega$ and $R_{P}=0.63 \mathrm{M} \Omega$, and $V_{t u r n-o n}=2.8 \mathrm{~V}, R_{S}=10.6 \Omega$ and $R_{P}=0.44 \mathrm{M} \Omega$, respectively. Compared to the forward $I-V$ characteristic, the reverse one has smaller turn-on voltage and series resistance (but similar shunt resistance), and hence a stronger light intensity is expected during the revere sweeping process. Figure $2 \mathrm{~d}$ shows the $E L$ intensity as a function of applied voltage for both forward (black curve) and reverse (red curve) sweeping processes. As expected, an optical hysteresis phenomenon is clearly observed due to the better reverse $I-V$ characteristic of the LEC. The observed electrical and optical hysteresis phenomena do not significantly affect the interband optical transitions in the $\mathrm{CsPbBr}_{3}$ QDs, as both the peak wavelength $\left(\lambda_{P} \sim 533 \mathrm{~nm}\right)$ and the spectral bandwidth (FWHM $\sim 16 \mathrm{~nm}$ ) obtained by the evolution of $E L$ spectra are quite stable regardless of the applied voltage (Figs. 2e, f). It suggests that the LEC is a reliable light source for the parallel reading process and is suitable to be integrated in the LEM structure.

We then perform the resistive switching characteristics of the $\mathrm{CsPbr}_{3}$ QD-based RRAM by applying a positive poling voltage on it, as shown in Fig. 3a. In contrast to the LEC, here the anode is the top Ag and the cathode corresponds to the bottom ITO electrodes. Ag, as the active metal, is easily oxidized and becomes to $\mathrm{Ag}^{+}$cation, as expressed by $\mathrm{Ag} \rightarrow \mathrm{Ag}^{+}+\mathrm{e}^{-}$ (left panel, Fig. 3a). The constant positive poling voltage further drifts $\mathrm{Ag}^{+}$cations to migrate over the entire $\mathrm{CsPbBr}_{3} \mathrm{QDs}$ active layer, and eventually reach to the bottom ITO cathode. $\mathrm{Ag}^{+}$cations are reduced back to neutral $\mathrm{Ag}$ atoms there, which serves as nucleation seeds for the subsequent growth of $\mathrm{Ag}$ clusters through repeated reduction processes of $\mathrm{Ag}^{+}$cations (middle panel, Fig. 3a). The Ag filament, which vertically connects the top and the bottom electrodes, is hence produced inside the active layer (right panel, Fig. 3a). A similar phenomenon has occurred for the bromide vacancy $\left(\mathrm{V}_{\mathrm{Br}}{ }^{+}\right)$, as it has been reported that most of the halide anions have low activation energy ${ }^{17,36,37}$, leading to facile migrations for both bromide anions $\left(\mathrm{Br}^{-}\right)$and bromide vacancy in the $\mathrm{CsPBr}_{3}$ QDs. When a positive poling voltage is applied on the RRAM, $\mathrm{V}_{\mathrm{Br}}{ }^{+}$is generated inside the active layer and then impelled towards the bottom ITO cathode. After capturing charge carriers there (as expressed by $\mathrm{V}_{\mathrm{Br}}{ }^{+}+$ $\mathrm{e}^{-} \rightarrow \mathrm{V}_{\mathrm{Br}}$ ), conducting channels are subsequently induced in a similar way as the formation of Ag filament. The processes for the formation of the $\mathrm{V}_{\mathrm{Br}}$ conducting channel and the $\mathrm{Ag}$ filament are reversible and responsible for the resistive switching effects in the RRAM, as will be discussed in more detail below. Figure $3 b$ shows the typical $I-V$ characteristic of the asfabricated RRAM by setting the compliance current to $I_{C C}=1 \mathrm{~mA}$. Accordingly, the RRAM exhibits a high electric resistance of $\sim 1.5 \times 10^{5} \Omega$ under the reading bias of $0.50 \mathrm{~V}$, and that represents the OFF state (or the HRS). During the positive voltage sweeping process from 0 to 2.5 and back to $0 \mathrm{~V}$ (or referred to as the SET process), the RRAM rapidly switches to the ON state (or the LRS) at $\sim 0.96 \mathrm{~V}$ with a low electric resistance down to $\sim 9.5 \times 10^{2} \Omega$ (constrained by $I_{C C}$ ), equivalent to two orders of 
magnitude of resistance plummeting. The device then switches back to the OFF state at $\sim-1.84 \mathrm{~V}$ while subject to a negative voltage sweeping process (from 0 to -2.5 to $0 \mathrm{~V}$ ), which is termed as the RESET process. In Fig. $3 \mathrm{c}$, we replot $I$ - $V$ characteristic of the RRAM in a $\log -\log$ scale [i.e., $\ln (I)$ vs. $\ln (V)]$ under both positive (top) and negative (bottom) voltage sweeping processes to elucidate the possible mechanism for the conduction behavior of OFF and ON states. The HRS for both sweeping modes can be interpreted by two regions, following the physical description of Ohm's law $(I \sim V)$ and space-charge-limited current (SCLC, also named as the Child law in which $I \sim V^{2}$ ) model. The observed transition from the Ohmic behavior to the SCLC indicates that the trap-unfilled regions are seized gradually by the charged carriers, leading to a larger current increment with the increased voltage. On the other hand, the conduction behavior of the LRS for both sweeping modes is well described by the Ohmic conduction accompanied by a dramatic reduction of electric resistance, indicating the $\mathrm{V}_{\mathrm{Br}}$ conducting channel and the Ag filament are well established inside the active layer in this stage. The ON/OFF current ratio, though declining from $\sim 10^{3}$ to $\sim 10^{2}$ after $5 \times 10^{3} \mathrm{~s}$, reflects the acceptable retention reliability of the $\mathrm{CsPbB}_{3}$ QD-based RRAM (Fig. 3d).

Next, we discuss the optical and electrical characteristics of the $\mathrm{CsPbBr}_{3}$ QD-based LEM. The bias voltage is applied to the top Ag electrode of the left device while that of the right device is grounded, so that the current flows from the left (right) to the right (left) during a positive (negative) voltage sweep. Figure 4a shows the variation of the $E L$ intensity (top panel) and the $I$ - $V$ characteristics (bottom panel) of the LEM when subject to a complete scanning cycle that includes both positive ( $0 \mathrm{~V} \rightarrow$ $8 \mathrm{~V} \rightarrow 0 \mathrm{~V})$ and negative $(0 \mathrm{~V} \rightarrow-8 \mathrm{~V} \rightarrow 0 \mathrm{~V})$ voltage sweeps. Similar to the typical RRAM, an initial electroforming process is needed to conduct the LEM for achieving the resistive switching effect (Fig. S3a, Supplementary Information). The electroforming voltage required for switching the LEM from HRS to LRS states is about -4.5 V, and a two-step hopping in the measured current is observed during the electroforming process, implying two different types of conducting channels induced in the left and right device of the LEM. During the positive voltage sweep, the EL emission is observed only from the right device of the LEM when the applied voltage exceeds $\sim 5 \mathrm{~V}$. The $E L$ emission appears only from the left device of the LEM upon switching to a negative voltage sweep, as seen by the photographs inserted in the top panel of Fig. 4 a. Clear optical hysteresis is again observed during both positive and negative voltage sweeps, due to the non-instantaneous response of the migrated ions to the applied voltage change as mentioned before.

The distinct behaviors of the $I$ - $V$ characteristics of the LEM observed in four different positive bias regions (denoted as (I), (II), (III), and (IV), respectively; see bottom panel of Fig. 4b) represent four different dynamic ionic transport and conduction processes: (I) $0-0.9 \mathrm{~V}$, where initial formation/reconstruction of the $\mathrm{Ag}$ and $\mathrm{V}_{\mathrm{Br}}$ filaments in the left device occur, (II) $0.9-1.9 \mathrm{~V}$, in which two ON-state RRAM devices are connected in series, (III) $1.9-4.6 \mathrm{~V}$, annihilation of the Ag filament and the rebuilding of $\mathrm{V}_{\mathrm{Br}}$ filament in the right device, and $(I V) 4.6-8.0 \mathrm{~V}$, the ON-state RRAM on the left is connected in 
series with the emitting LEC on the right. Possible scenarios of ion migrations in each bias region are plotted in Fig. 4b, with the corresponding band diagrams of the LEM operating in these regions shown in Fig. S4. After the electroforming process, both the Ag filament and $\mathrm{V}_{\mathrm{Br}}$ conducting channel are well established in the right device, whereas only the $\mathrm{V}_{\mathrm{Br}}$ conducting channel is induced on the left (Fig. S3b, Supplementary Information). A positive voltage in the range of $0-0.9 \mathrm{~V}$ erases the original $\mathrm{V}_{\mathrm{Br}}$ conducting channel of the left device through the oxidation process $\left(\mathrm{V}_{\mathrm{Br}} \rightarrow \mathrm{V}_{\mathrm{Br}}{ }^{+}+\mathrm{e}^{-}\right)$, without affecting the high conductivity of the right. The continuous supplement of positive voltage facilitates the gradual formation of Ag filament initiating at the bottom ITO electrode in the left device via the reduction process of $\mathrm{Ag}^{+}$cations. Meanwhile, a new $\mathrm{V}_{\mathrm{Br}}$ conducting channel is also rebuilt (also from the bottom to the top electrode) through a similar reduction process $\left(\mathrm{V}_{\mathrm{Br}}{ }^{+}+\mathrm{e}^{-} \rightarrow\right.$ $\mathrm{V}_{\mathrm{Br}}$ ). The current measured in region $(I)$ is hence increased rapidly from $\sim 1 \times 10^{-7}$ to $\sim 2 \times 10^{-5} \mathrm{~mA}$ with the bias voltage, with the maximum current in this region restricted by the left device as its resistance is much larger than that of the right (i.e., equivalent to an $\mathrm{ON}$-state RRAM in this stage).

While further increasing the applied voltage to phase into region (II), the LEM current is suddenly switched and significantly increased to $\sim 4 \times 10^{-3} \mathrm{~mA}$, results from the complete formation of the Ag filament and the reconstruction of $\mathrm{V}_{\mathrm{Br}}$ conducting channel in the left device. Here, each device on either side of the LEM functions as the ON-state RRAM and they are connected in series, rendering the maximum current (or the lowest resistance) of the LEM. Further ramping up the positive voltage to region (III) cause both the $\mathrm{Ag}$ filament and the $\mathrm{V}_{\mathrm{Br}}$ conducting channel in the right device start vanishing due to their associated oxidation processes, leading to an obvious current drop back down to $\sim 2 \times 10^{-5} \mathrm{~mA}$. Here shall be addressed that another $\mathrm{V}_{\mathrm{Br}}$ conducting channel can be induced and rebuilt (from the top to the bottom electrode) in the right device during this stage. Finally, a well rectifying diode-like behavior with a turn-on voltage of $\sim 5 \mathrm{~V}$ is observed when the positive voltage reaches region $(I V)$, in which $\mathrm{Cs}^{+}$cations could be dissociated from the bromide vacancy as previous calculations showed that $\mathrm{Cs}^{+}$cation has a much higher activation energy than other halide anions ${ }^{36,37}$. The respective repelling of $\mathrm{Cs}^{+}$cations and $\mathrm{Br}^{-}$anions towards the top $\mathrm{Ag}$ electrode and the bottom ITO results in the formation of p-i-n homojunction in the right device (while the left device remains as an ON-state RRAM), responsible for the subsequent $E L$ emission observed in the LEM. The LEM has no EL emissions until the applied voltage reaches $\sim 5 \mathrm{~V}$, which is equivalent to the sum of the turnon voltage of the individual LEC ( 4.0 V, Fig. 2b) and the RRAM ( 1.0 V, Fig. 3b), and that is reasonable when considering both devices are connected in series. As far as conversion efficiency and energy saving are concerned, optimization of the device fabrication such as better control of the $\mathrm{CsPbr}_{3}$ QDs thin-film thickness and the employment of a new device scheme could further reduce the LEM turn-on voltage. When the applied voltage is swept back from 8 to $0 \mathrm{~V}$, a similar electrical hysteresis effect is clearly observed (in reference to the previous discussion of Fig. 2c), validating that in region (IV), the LEM 
is realized with the ON-state RRAM on the left connecting in series to the emitting LEC on the right. As a result, the proposed LEM can carry out an additional parallel and non-contact optical reading through detecting the EL emission of the LEC, besides the electrical reading proceeded in the RRAM. The negative part of the $I-V$ characteristics exhibits the same behavior as the positive voltage, which a is easily understood by the symmetry of the LEM device structure. This enables one to modulate the functionality of each device in the LEM to be either as RRAM or LEC by simple switching the sweep polarity applied to the LEM.

Figure 5a shows the variation of EL intensity of the LEM versus detecting time under a modulated bias with a repetitive sequence of $8 \mathrm{~V} / 0 \mathrm{~V} /-8 \mathrm{~V} / 0 \mathrm{~V}$. At each bias, the applied voltage remains for 2 seconds before switching to other bias values to acquire a stable $E L$ intensity. When a positive voltage of $8 \mathrm{~V}$ is applied to the LEM, the detected $E L$ intensity (plotted in red curves of Fig. 5a) is attributed to the EL emission from the right device of the LEM (as the previous discussion related to region (IV) of Fig. 4b). The EL emission emerges from the left device while changing the electric voltage to $-8 \mathrm{~V}$, and the $E L$ intensity recorded for this case is plotted as blue curves in Fig. 5a. The inserted photographs show the emission images under the bias voltages of $8 \mathrm{~V}$ (left) and $-8 \mathrm{~V}$ (right). The observed switching of the $E L$ emission in between the right and the left devices of the LEM follows the polarity exchange of the bias voltage, and no light is detected as soon as the LEM is turned off. Figure 5b plots the corresponding variation of the LEM current versus detecting time under the same modulated bias condition as that for measuring the EL intensity, where the red and blue curves represent the data collected under the bias voltage of $8 \mathrm{~V}$ and $-8 \mathrm{~V}$, respectively. It can be seen that the extremum currents obtained in the LEM is $\sim \pm 15 \mathrm{~mA}$ for the bias voltages of $\pm 8 \mathrm{~V}$, which is consistent with the symmetrical $I-V$ characteristics as shown in Fig. 4a. The results in Figs. 5a and $5 \mathrm{~b}$ validate that the $E L$ emission can indeed be switched from the device on either side of the LEM, which not only allows the simultaneous optical and electrical readings of the encoded information but also enables modulation at the same time, significantly extending the scope of the application of our LEM. Figure 5c shows the retention time of the LEM by continuously reading out its $E L$ intensity $(L)$ and electric current $(I)$ under the bias voltages of $\pm 8 \mathrm{~V}$. The current decreases from $I=21.4$ to $15.4 \mathrm{~mA}$ under $8 \mathrm{~V}$ after 3600 seconds (red circles, top panel), whereas the $E L$ intensity falls to $\sim 70 \%$ of the initial value (red square, top panel). While the bias condition is switched to $-8 \mathrm{~V}$ (bottom panel), similar retention behaviors are observed for both $E L$ intensity and current. It suggests the optical and electrical signals of the LEM are nearly stable, even the functionality of the separate device is completely changed after switching the voltage polarity applied to the LEM.

\section{Conclusions}


In conclusion, a new $\mathrm{CsPbBr}_{3}$ QD-based perovskite LEM device with reliable modulation and acceptable retention is demonstrated capable of reading out the encrypted information by simultaneously detecting its optical and electrical signals. We believe this work can pave the way towards a new design for the advanced perovskite-based devices addressing high integration and easy modulation between optical communication and electrical transmission processes.

\section{Methods}

Synthesis of $\mathrm{CsPbBr}_{3}$ QDs

Lead (II) bromide $\left(\mathrm{PbBr}_{2}, 0.1845 \mathrm{~g}\right)$ and cesium bromide (CsBr, $\left.0.1415 \mathrm{~g}\right)$ powders were dissolved in dimethyl sulfoxide (DMSO, $5 \mathrm{~mL}$ ) to form the precursor solution. Oleic acid (OA, $0.5 \mathrm{~mL})$, oleylamine (OAm, $0.25 \mathrm{~mL})$, and hydrogen bromide $(\mathrm{HBr}, 5 \mu \mathrm{L})$ were then added to stabilize the precursor solution and kept at a stirring rate of $1000 \mathrm{rpm}$ for $1 \mathrm{hour}$. The mixed precursor solution was then poured into toluene $(240 \mathrm{~mL})$ to trigger the supersaturated recrystallization process for synthesizing the $\mathrm{CsPbBr}_{3}$ QDs. After that, the entire solution is centrifuged at $3000 \mathrm{rpm}$ for 5 seconds and we take its supernatant part by using a pipette to filter out the large particles. All of the above were conducted at room temperature.

\section{Deposition of Ag and ITO electrodes}

The top Ag $(100 \mathrm{~nm})$ and the bottom ITO $(250 \mathrm{~nm})$ electrodes were grown by using a RF sputtering system with the background chamber pressure evacuated to of $\sim 1.0 \times 10^{-6}$ torr. The working pressure and sputtering power were kept at $\sim 8.0 \times 10^{-3}$ torr and $50 \mathrm{~W}$, respectively. The inert gas of argon with a flux of 6.0 and $10.0 \mathrm{sccm}$ was introduced into the sputtering chamber while depositing Ag and ITO films, respectively. The deposition rate was 4.0 $\AA$ /s for both Ag and ITO films.

\section{Measurement Details}

EL spectra and intensities of the fabricated sample were measured by using a portable spectrometer (Ocean Optics USB4000), while its electric characterizations were performed with a Keithley 2400 source meter. XRD patterns were measured by a multipurpose X-ray diffraction system (Bruker new D8 discover). Absorption spectra of the fabricated samples were obtained by the spectrophotometer (Aglient, Cary5000), and the PL spectra were measured by a portable spectrometer (Ocean Optics USB4000) with a $405 \mathrm{~nm}$ diode laser excitation. Surface and cross-sectional morphologies of the fabricated samples were examined using field-emission SEM (JEOL, JSM7600F, $10 \mathrm{kV}$ ). TEM images and EDS spectra of the as-synthesized $C s \mathrm{PbBr}_{3}$ QDs were performed by using an atomic-resolution electron microscope (JEOL, ARM200F, $200 \mathrm{kV}$ ).

\section{Data availability}


All data supporting this study and its findings are available within the article and its Supplementary Information or from the corresponding author upon reasonable request.

\section{Code availability}

The codes that support the findings of this study are available from the corresponding author upon reasonable request.

\section{References}

1. Chang, T. C., Chang, K. C., Tsai, T. M., Chu, T. J. \& Sze, S. M. Resistance random access memory. Materials Today 19, 254-264 (2016).

2. Wuttig, M., Bhaskaran, H. \& Taubner, T. Phase-change materials for non-volatile photonic applications. Nat. Photon 11, 465-476 (2017).

3. Zhao, X., Xu, H., Wang, Z., Lin, Y. \& Liu, Y. Memristors with organic-inorganic halide perovskites. InfoMat 1, 183210 (2019).

4. Tseng, R. J., Ouyang, J., Chu, C. W., Huang, J. \& Yang, Y. Nanoparticle-induced negative differential resistance and memory effect in polymer bistable light-emitting device. Appl. Phys. Lett. 88, 123506 (2006).

5. Zakhidov, A. A., Jung, B., Slinker, J. D., Abruña, H. D. \& Malliaras, G. G. A light-emitting memristor. Org. Electron. 11, 150-153 (2010).

6. Chang, C. W., Tan, W. C., Lu, M. L., Pan, T. C., Yang, Y. J. \& Chen, Y. F. Electrically and optically readable light emitting memories. Sci. Rep. 4, 5121 (2014).

7. Liou, Y. R., Haider, G., Cai, S. Y., Wu, C. L., Lin, T. Y. \& Chen, Y. F. High-Performance Light-Emitting Memories: Multifunctional Devices for Unveiling Information by Optical and Electrical Detection. Adv. Opt. Mater. 4, 1744-1749 (2016).

8. Anutgan, T., Anutgan, M., Atilgan, I. \& Katircioglu, B. Electroformed silicon nitride based light emitting memory device. Appl. Phys. Lett. 111, 053502 (2017).

9. Zhang, Y. et al. Broadband transparent optical phase change materials for high-performance nonvolatile photonics. Nat. Commun. 10, 4279 (2019).

10. Bera, A., Peng, H., Lourembam, J., Shen, Y., Sun, X. W. \& Wu, T. A Versatile Light-Switchable Nanorod Memory: Wurtzite ZnO on Perovskite SrTiO3. Adv. Funct. Mater. 23, 4977-4984 (2013).

11. Tan, H. et al. Light-gated memristor with integrated logic and memory functions. ACS Nano 11, 11298-11305 (2017).

12. Chen, J. Y., Chiu, Y. C., Li, Y. T., Chueh, C. C. \& Chen, W. C. Nonvolatile perovskite-based photomemory with a multilevel memory behavior. Adv. Mater. 29, 1702217 (2017).

13. Ríos, Carlos et al. In-memory computing on a photonic platform. Sci. Adv. 5: eaau5759 (2019).

14. Yakunin, S. et al. Low-threshold amplified spontaneous emission and lasing from colloidal nanocrystals of caesium lead halide perovskites. Nat. Commun. 6, 1-9 (2015).

15. Leng, J. et al. Thermodynamic Control in the Synthesis of Quantum-Confined Blue-Emitting CsPbBr3 Perovskite Nanostrips. J. Phys. Chem. Lett. 11, 2036-2043 (2020). 
16. Xiao, Z. et al. Giant switchable photovoltaic effect in organometal trihalide perovskite devices. Nat. Mater. 14, 193-198 (2015).

17. Eames, C., Frost, J. M., Barnes, P. R., O’regan, B. C., Walsh, A. \& Islam, M. S. Ionic transport in hybrid lead iodide perovskite solar cells. Nat. Mater. 6, 1-8 (2015).

18. Chen, H. W., Sakai, N., Ikegami, M. \& Miyasaka, T. Emergence of hysteresis and transient ferroelectric response in organo-lead halide perovskite solar cells. J. Phys. Chem. Lett. 6, 164-169 (2015).

19. Meloni, S. et al. Ionic polarization-induced current-voltage hysteresis in $\mathrm{CH}_{3} \mathrm{NH}_{3} \mathrm{PbX}_{3}$ perovskite solar cells. Nat. Commun. 7, 1-9 (2016).

20. Li, J., Bade, S. G. R., Shan, X. \& Yu, Z. Single-layer light-emitting diodes using organometal halide perovskite/poly (ethylene oxide) composite thin films. Adv. Mater. 27, 5196-5202 (2015).

21. Zhang, H. et al. Organic-inorganic perovskite light-emitting electrochemical cells with a large capacitance. Adv. Funct. Mater. 25, 7226-7232 (2015).

22. Alahbakhshi, M. et al. Bright and effectual perovskite light-emitting electrochemical cells leveraging ionic additives. ACS Energy Lett. 4, 2922-2928 (2019).

23. Wang, Y. et al. Synergies of Electrochemical Metallization and Valance Change in All-Inorganic Perovskite Quantum Dots for Resistive Switching. Adv. Mater. 30, 1800327 (2018).

24. Zhu, X., Lee, J. \& Lu, W. D. Iodine vacancy redistribution in organic-inorganic halide perovskite films and resistive switching effects. Adv. Mater. 29, 1700527 (2017).

25. Gu, C. \& Lee, J. S. Flexible hybrid organic-inorganic perovskite memory. ACS Nano 10, 5413-5418 (2016).

26. Liu, D. et al. Flexible all-inorganic perovskite $\mathrm{CsPbBr}_{3}$ nonvolatile memory device. ACS Appl. Mater. Interfaces 9, 61716176 (2017).

27. Luo, W., Wu, C., Sun, W., Guo, X., Xiao, L. \& Chen, Z. High crystallization of perovskite film by a fast electric current annealing process. ACS Appl. Mater. Interfaces 9, 26915-26920 (2017).

28. Liang, P. et al. Unusual Stability and Temperature-Dependent Properties of Highly Emissive CsPbBr3 Perovskite Nanocrystals Obtained from in Situ Crystallization in Poly (vinylidene difluoride). ACS Appl. Mater. Interfaces 11, 22786-22793 (2019).

29. Yang, K., Cho, K., Kim, S. \& Im, K. Influence of thermal stress on heat-generating performance of indium tin oxide nanoparticle thin films. J. Vac. Sci. Technol. B 34, 06KA02 (2016).

30. Hamasha, M. M. et al. Stability of ITO thin film on flexible substrate under thermal aging and thermal cycling conditions. J. Display Technol. 8, 385-390 (2012).

31. Wang, H. \& Yan, X. Overview of resistive random access memory (RRAM): Materials, filament mechanisms, performance optimization, and prospects. Phys. Status Solidi RRL 13, 1900073 (2019).

32. Hoffman, J. B., Zaiats, G., Wappes, I. \& Kamat, P. V. CsPbBr 3 solar cells: controlled film growth through layer-by-layer quantum dot deposition. Chem. Mater. 29, 9767-9774 (2017).

33. X. Zhang, Y. Qian, X. Ling, Y. Wang, Y. Zhang, J. Shi, Y. Shi, J. Yuan, W. Ma, ACS Appl. Mater. Interfaces 2020, 12, 27307.

34. Lee, Y. J., Lee, C. J. \& Chen, C. H. Effect of surface texture and backside patterned reflector on the AlGaInP lightemitting diode: high extraction of waveguided light. IEEE J. Quantum Electron. 47, 636-641 (2011). 
35. Lee, Y. J., Lee, C. J. \& Chen, C. H. Determination of junction temperature in InGaN and AlGaInP light-emitting diodes. IEEE J. Quantum Electron. 46, 1450-1455 (2010).

36. Pan, D., Fu, Y., Chen, J., Czech, K. J., Wright, J. C. \& Jin, S. Visualization and studies of ion-diffusion kinetics in cesium lead bromide perovskite nanowires. Nano Lett.18, 1807-1813 (2018).

37. Lai, M. et al. Intrinsic anion diffusivity in lead halide perovskites is facilitated by a soft lattice. PNAS 115, 11929-11934 (2018).

\section{Acknowledgements}

C.-C.C. and Y.-J. L. acknowledge the financial support from the Ministry of Science and Technology in Taiwan (contract Nos. MOST 108-2112-M-003-015-MY3 and MOST 109-2112-M-003-014-MY3). J.L. and K.T acknowledge the support of JSPS KAKENHI (Grant No. 19H05627).

\section{Author Information}

Affiliations

Institute of Electro-Optical Engineering, National Taiwan Normal University, Taipei, 11677, Taiwan

Ya-Ju Lee, Kang-Hsiang Liu Yi Peng, Meng-Cheng Yen \& Chun-Chieh Chang

Institute for Materials Chemistry and Engineering (IMCE), Kyushu University, 744 Motooka, Nishiku, Fukuoka 819-0395, Japan

Junfu Leng \& Kaoru Tamada

Contributions

Y.J.L. conceived the idea. Y.J.L. and C.C.C. designed the experiments. K.H.L. and Y.P. conducted device fabrication. K.H.L., Y.P., M.C.Y. and J. L. assisted with the experiments and performed the measurements. Y.J.L., K.H.L., and C.C.C. did data analysis. Y.J.L. and C.C.C. drafted the manuscript. Y.J.L., C.C.C. and K.T. supervised this work. All the authors discussed the results and commented on the manuscript.

Corresponding authors

Correspondence to: Ya-Ju Lee or Chun-Chieh Chang or Kaoru Tamada

\section{Ethics declarations}

The authors declare no competing financial interests. 
a

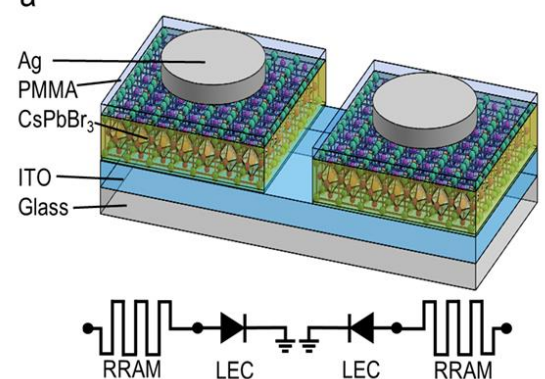

d

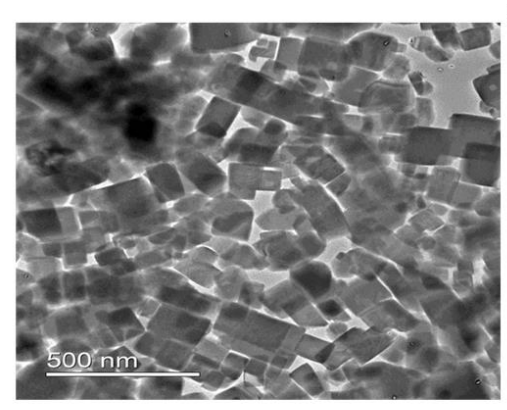

b
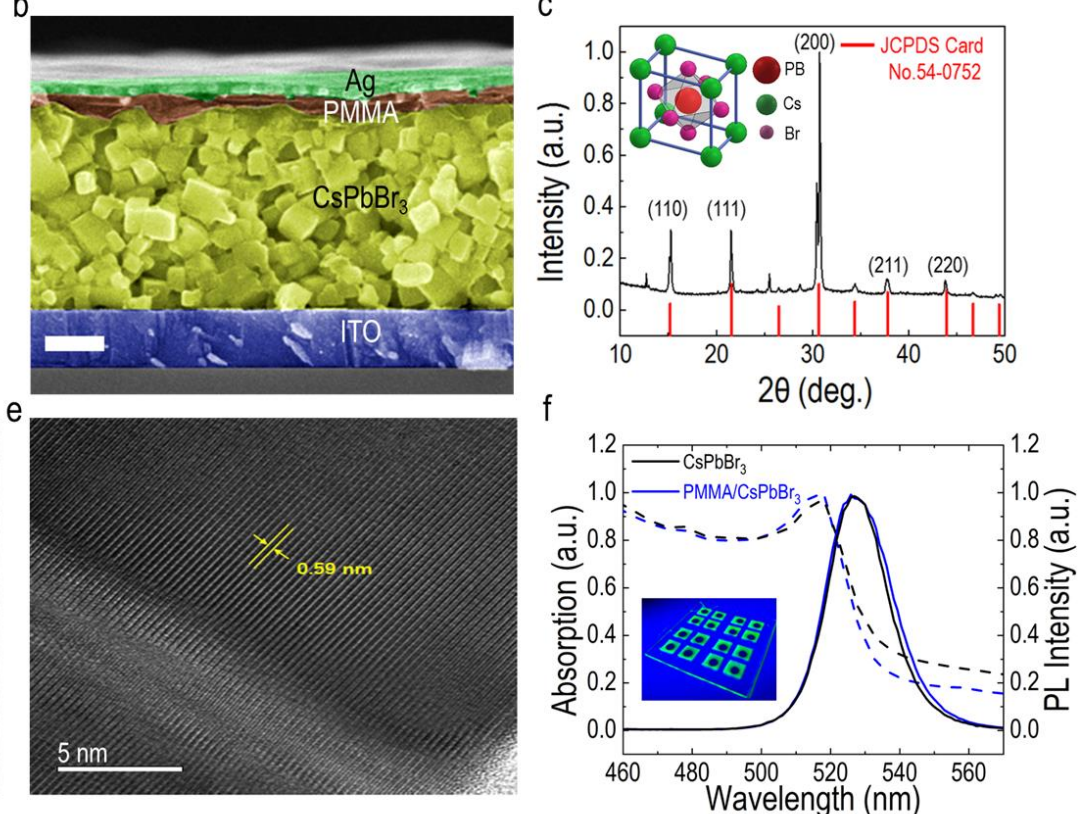

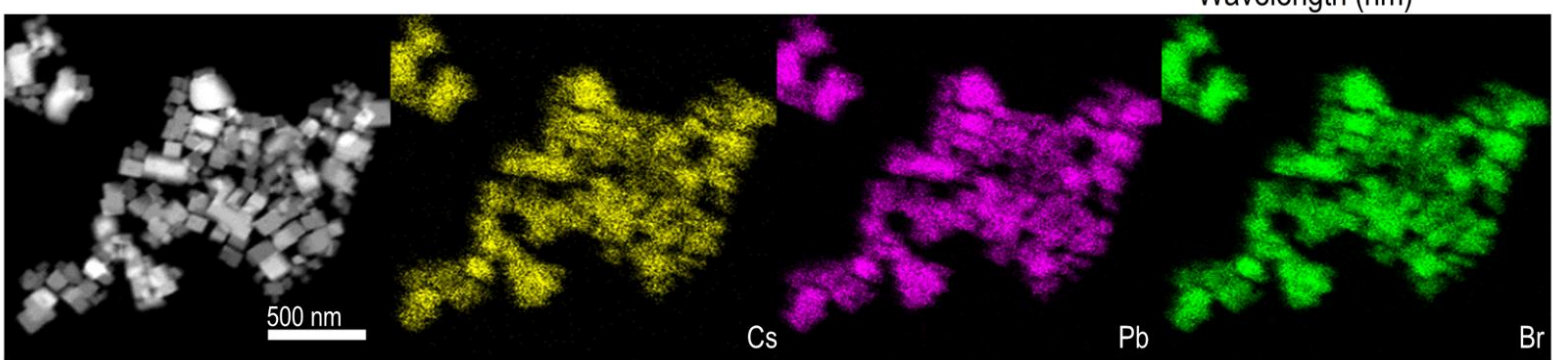

Figure 1 a Schematic of the $\mathrm{CsPbr}_{3}$ QDs-based LEM device composed of two nominally identical devices (top plot). Effective electronic circuits illustrating each device has dual functionalities either as RRAM or LEC, depending on the electric field direction across the LEM (bottom plot). b Cross-sectional scanning electron microscope (SEM) image of the as-fabricated LEM, where the scale bar is $250 \mathrm{~nm}$. $\mathbf{c}$ XRD pattern of the $\mathrm{CsPbBr}_{3}$ QDs along with that of the cubic structure (JSPDS No. 540752). A schematic crystal structure view of $\mathrm{CsPbBr}_{3}$ is inserted in the figure. d TEM image of the $\mathrm{CsPbBr}_{3}$ QDs. e High resolution of the $\mathrm{CsPbBr}_{3}$ QDs to identify the interplanar space of (200) plane. A clear fused interface is also observed in this figure. f Absorption (primary vertical axis) and PL spectra (secondary vertical axis) of the $\mathrm{CsPbr}_{3}$ QDs quasi-continuous film with and without the PMMA protection layer. Inset: A photograph of the as-fabricated LEM taken under the illumination of $405 \mathrm{~nm}$ UV-LED. g EDX mapping of the $\mathrm{CsPbBr}_{3}$ QDs for analyzing the elements of $\mathrm{Cs}, \mathrm{Pb}$, and $\mathrm{Br}$. 
a

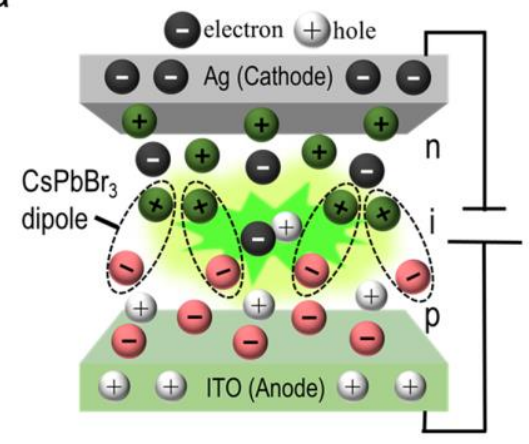

d

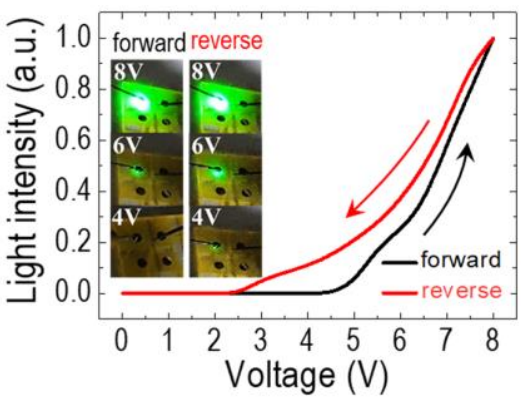

b

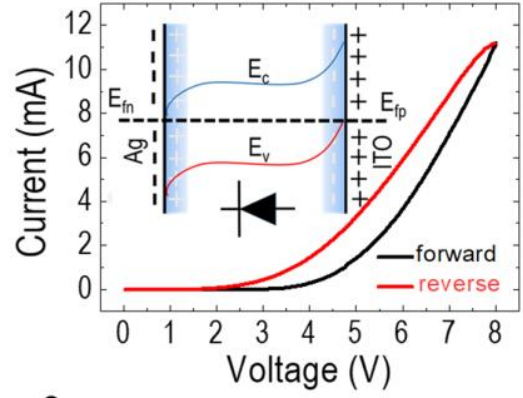

e

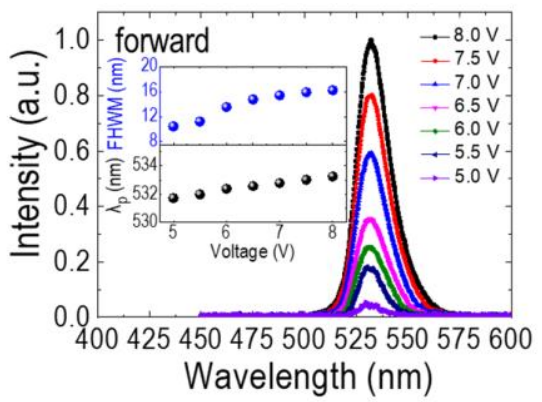

C

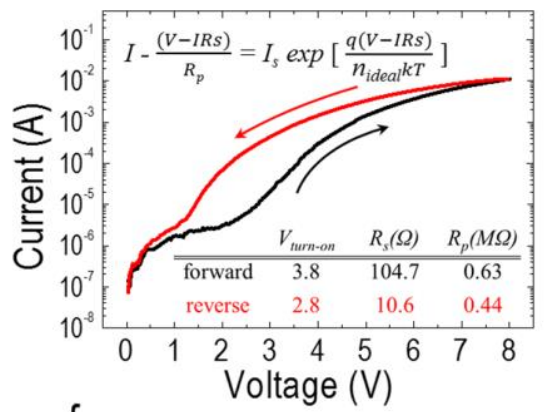

f

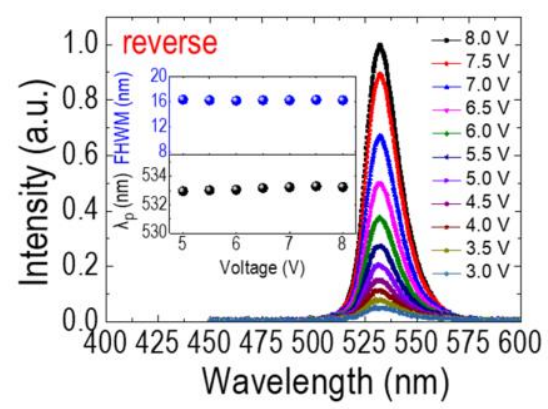

Figure 2 a Schematic illustration of ion migration inducing electric dipoles aligning along the direction of an applied electric field. Cations and anions are hence accumulated close to the Ag and ITO electrodes, respectively, leading to the formation of p-i-n diode (bottom-up). b $I$ - $V$ characteristics of the perovskite LEC for the sweep voltage range of $0 \mathrm{~V} \rightarrow 8 \mathrm{~V} \rightarrow 0 \mathrm{~V}$, and a clear hysteresis effect is observed in this figure. Inset: the corresponding energy-level diagram while applying a negative poling voltage on the LEC (Ag: cathode; ITO: anode). c Re-plotting I- $V$ characteristics of the perovskite LEC in a semi-log scale. Insets: the modified Shockley equation (top) and a summary table of the turn-on voltage, series and shunt resistances extracted from the forward and reverse $I-V$ characteristics (bottom). $\mathbf{d} E L$ intensity of the perovskite LEC versus sweeping voltage in a range of $0 \mathrm{~V} \rightarrow 8 \mathrm{~V} \rightarrow 0 \mathrm{~V}$. Insets show emission images of the LEC under forward (left) and reverse (right) voltages of 4, 6, and $8 \mathrm{~V}$. Evolutions of $E L$ spectra with the increase of $\mathbf{e}$ forward and $\mathbf{f}$ reverse voltages. Variation trends of the peak wavelength $\left(\lambda_{\mathrm{P}}\right)$ and the spectral bandwidth (FWHM) for the $E L$ spectra with the increasing voltage are also inserted in both figures. 

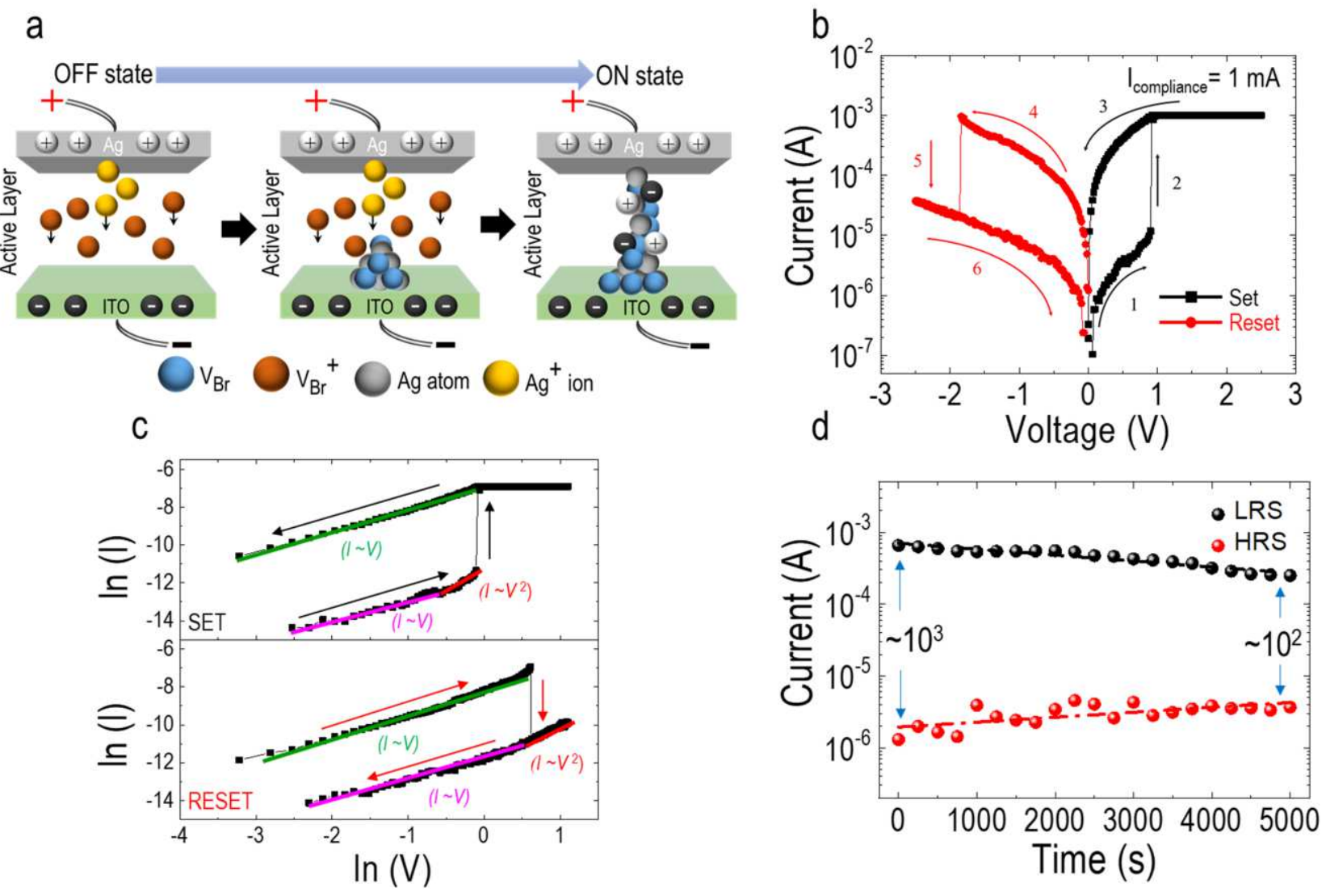

Figure 3 a Illustration of resistive switching effect in the $\mathrm{CsPBBr}_{3}$ QD-based RRAM at the initial stage (left), during the migration of $\mathrm{Ag}^{+}$cations and $\mathrm{V}_{\mathrm{Br}}{ }^{+}$vacancies (middle), and the final formation of the $\mathrm{V}_{\mathrm{Br}}$ conducting channel and the $\mathrm{Ag}$ filament (right). b Typical $I-V$ characteristic of the $\mathrm{CsPbBr}_{3}$ QD-based RRAM by setting the compliance current to $I_{C C}=1 \mathrm{~mA}$. $\mathbf{c}$ Replotted $I-V$ characteristic of the $\mathrm{CsPbBr}_{3}$ QD-based RRAM in a log-log scale with fitted conduction mechanisms (pink and red lines represent linear Ohmic and quadratic space-charge-limited conductions in HRS, respectively, whereas green line represents linear Ohmic conduction in LRS) for SET (top) and RESET (bottom) conditions. $\mathbf{d}$ Retention characteristics of LRS and HRS states. Reading bias is set to $0.3 \mathrm{~V}$ in this measurement. 
a

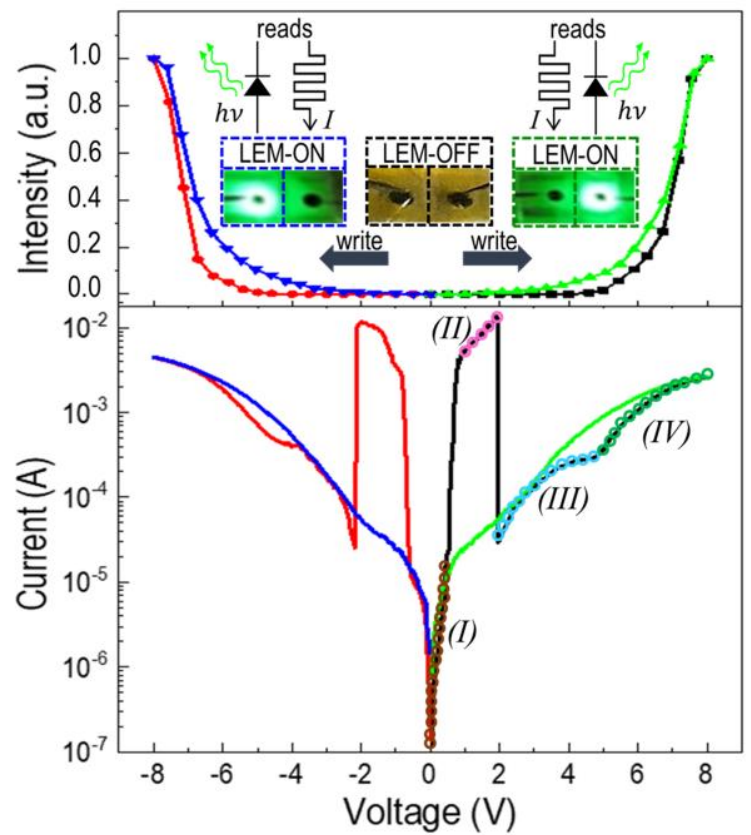

b
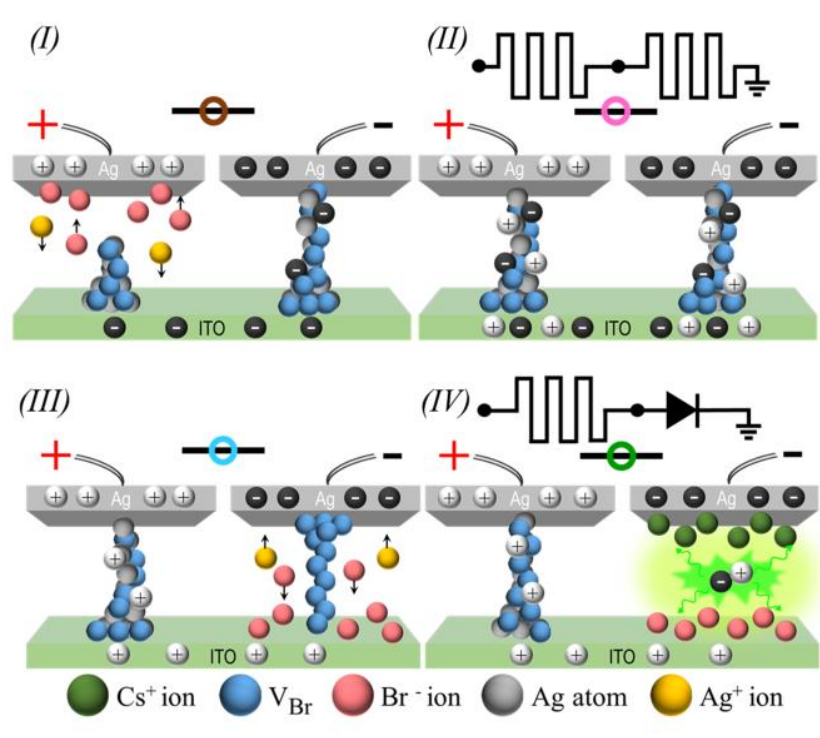

Figure 4 a Variations of $E L$ intensity (top) and $I$ - $V$ characteristic (bottom) of the LEM during subjecting to a complete scanning cycle including both positive $(0 \mathrm{~V} \rightarrow 8 \mathrm{~V} \rightarrow 0 \mathrm{~V})$ and negative $(0 \mathrm{~V} \rightarrow-8 \mathrm{~V} \rightarrow 0 \mathrm{~V})$ voltage sweeps. The positive part of the $I-V$ characteristic is divided into four regions to clarify the underlying mechanism for the observed optoelectronic characteristics of the $\mathrm{CsPbBr}_{3}$ QD-based LEM. Insets: photographs taken while applying $0 \mathrm{~V}$ (middle), $8 \mathrm{~V}$ (right), and -8 V(left) to the LEM, which addressing the LEM's potential of enabling synchronous optical and electrical readings with high modulation ability. $\mathbf{b}$ Possible scenarios of ions migrations in accordance with individual region of $(I)-(I V)$. 

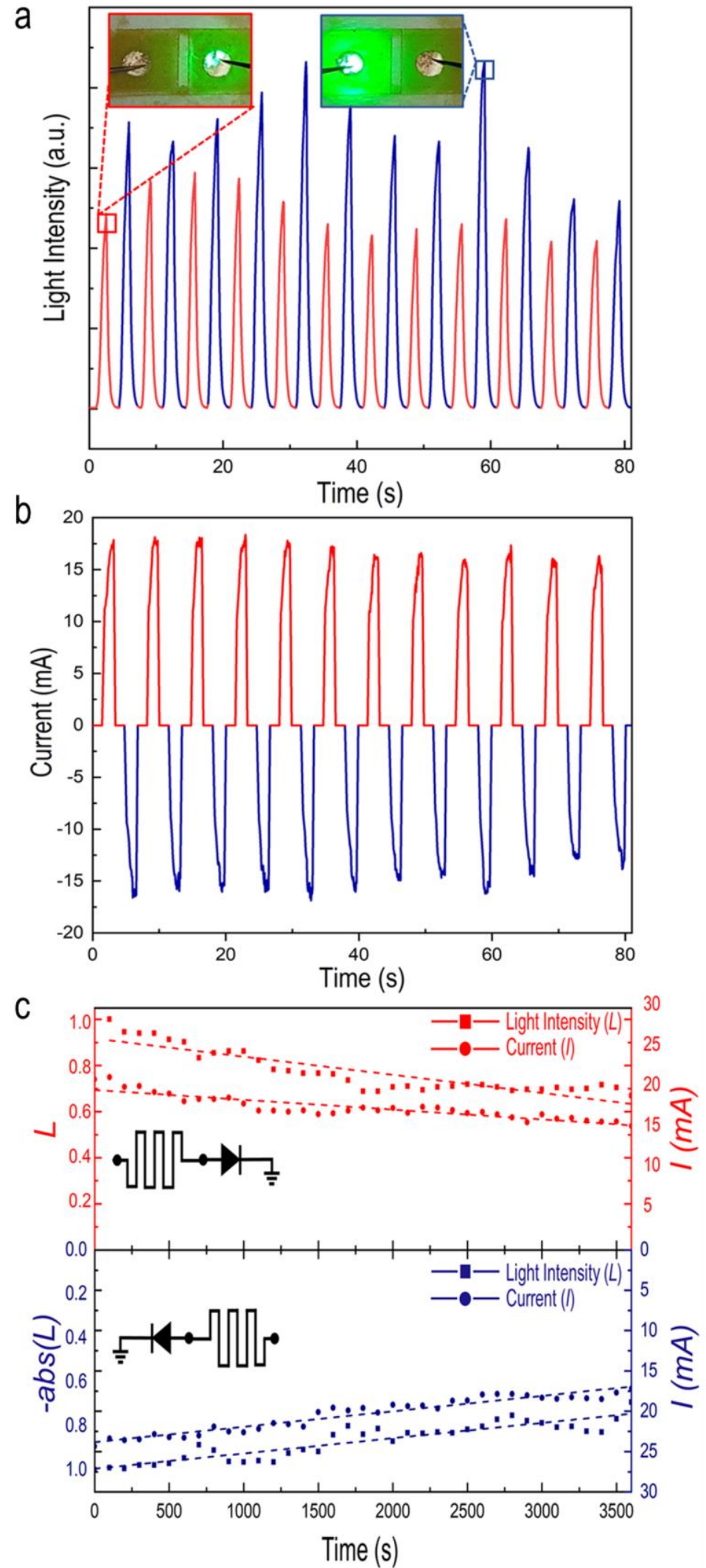
Figure 5 a Variations of LEM EL intensity versus detecting time under a modulated bias with a repetitive sequence of 8 V/ 0 $\mathrm{V} /-8 \mathrm{~V} / 0 \mathrm{~V}$. Insets: photographs of emission images under different bias voltages of $8 \mathrm{~V}$ (left) and $-8 \mathrm{~V}$ (right) applied to the LEM. b Corresponding variation of the LEM electric current versus detecting time under the same modulated bias condition as a. c Retention characteristics by continuously reading out the $E L$ intensity $(L)$ and electric current $(I)$ under applying bias voltages of $8 \mathrm{~V}$ (top) and $-8 \mathrm{~V}$ (bottom) to the LEM. The effective electrical circuits of the LEM are also plotted for different bias circumstances. 


\section{Figures}

a

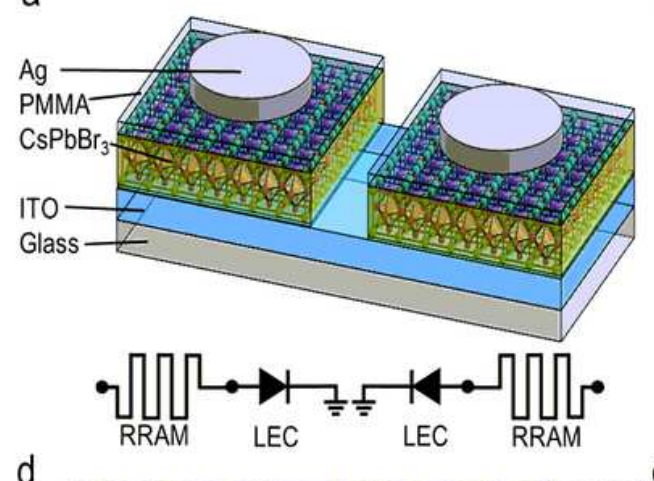

d

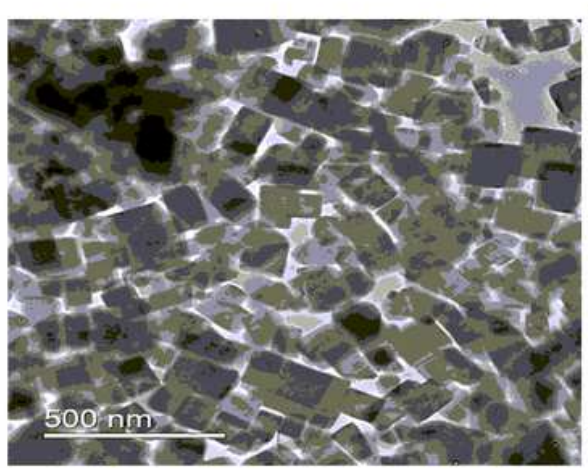

b

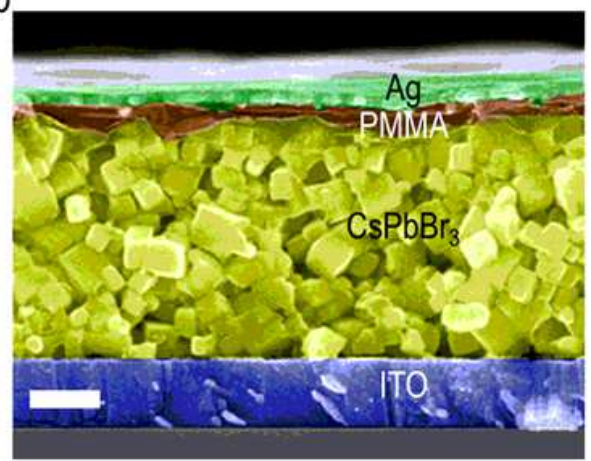

e

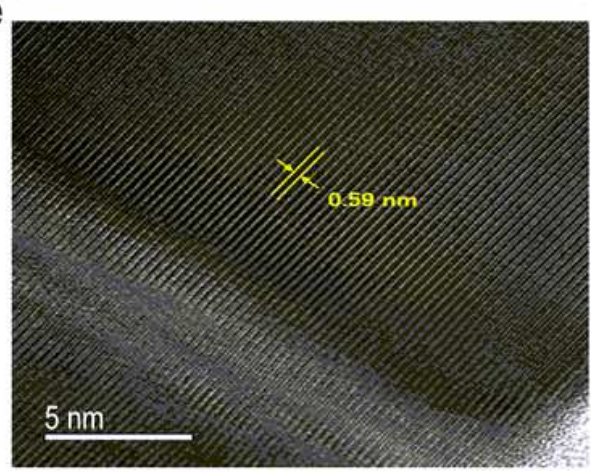

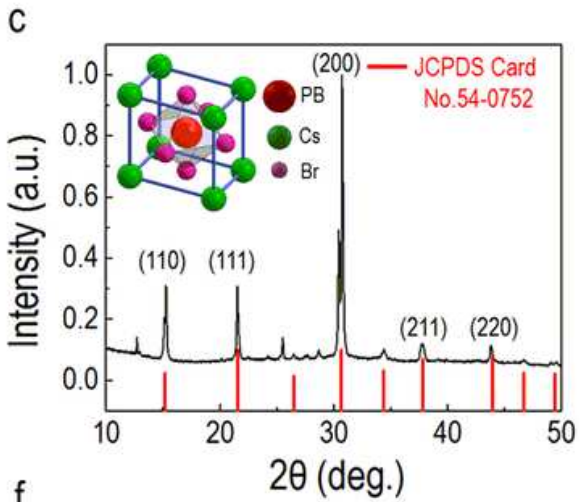

$f$

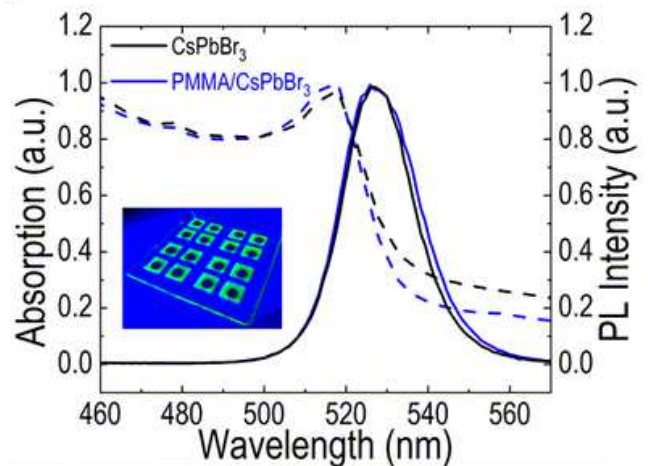

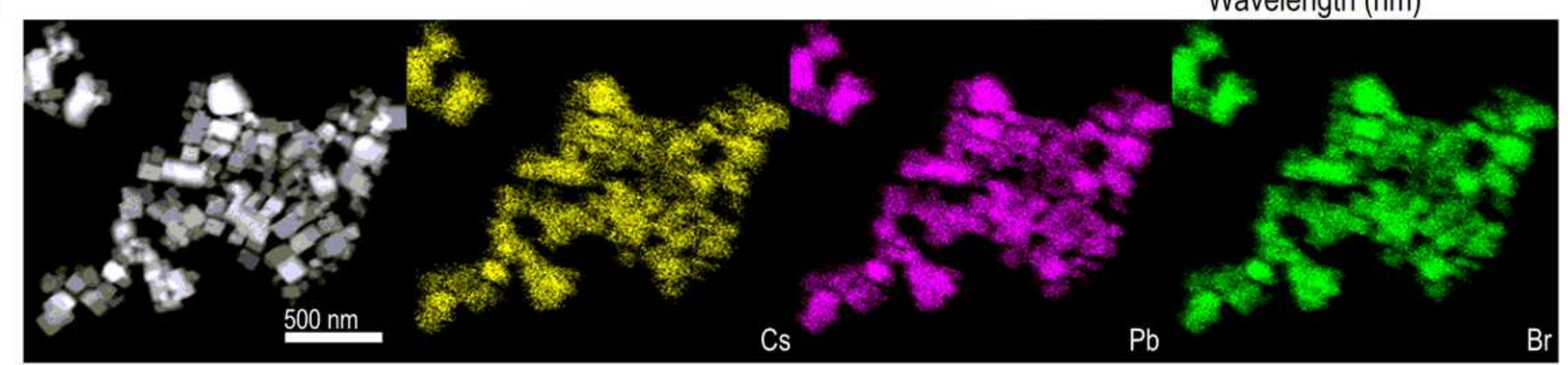

Figure 1

a Schematic of the CsPbBr3 QDs-based LEM device composed of two nominally identical devices (top plot). Effective electronic circuits illustrating each device has dual functionalities either as RRAM or LEC, depending on the electric field direction across the LEM (bottom plot). b Cross-sectional scanning electron microscope (SEM) image of the as-fabricated LEM, where the scale bar is $250 \mathrm{~nm}$. c XRD pattern of the CsPbBr3 QDs along with that of the cubic structure (JSPDS No. 54-0752). A schematic crystal structure view of $\mathrm{CsPbBr} 3$ is inserted in the figure. $\mathrm{d}$ TEM image of the $\mathrm{CsPbBr} 3$ QDs. e High resolution of the CsPbBr3 QDs to identify the interplanar space of (200) plane. A clear fused interface is also observed in this figure. f Absorption (primary vertical axis) and PL spectra (secondary vertical axis) of the $\mathrm{CsPbBr} 3$ QDs quasi-continuous film with and without the PMMA protection layer. Inset: A photograph of the asfabricated LEM taken under the illumination of $405 \mathrm{~nm}$ UV-LED. $g$ EDX mapping of the CsPbBr3 QDs for analyzing the elements of $\mathrm{Cs}, \mathrm{Pb}$, and $\mathrm{Br}$. 
a

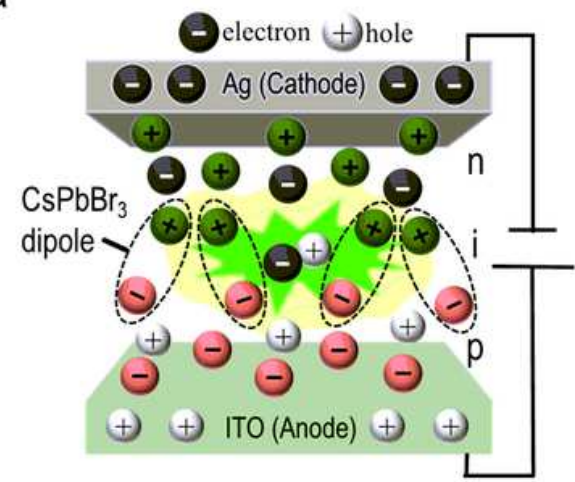

d

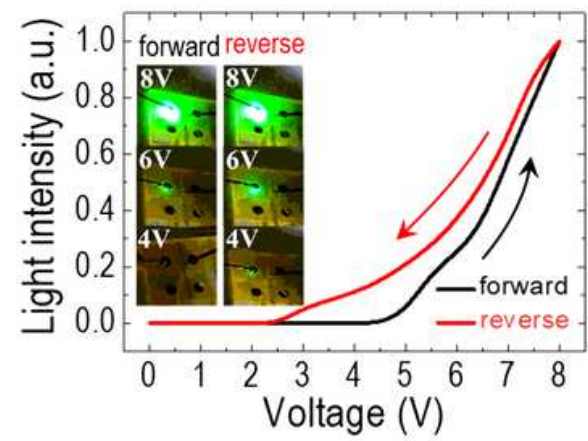

b

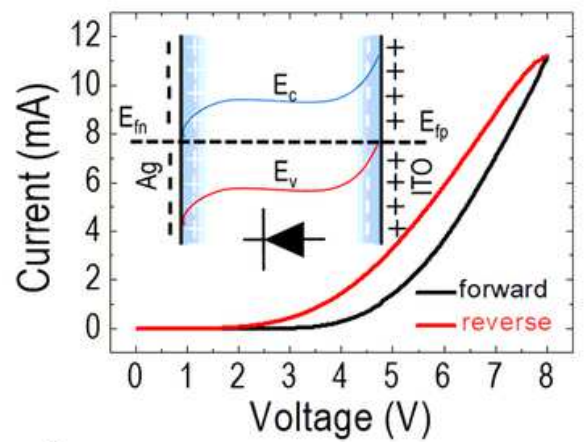

e

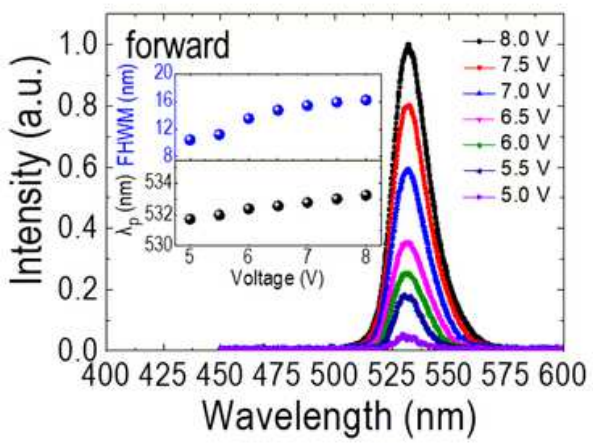

C

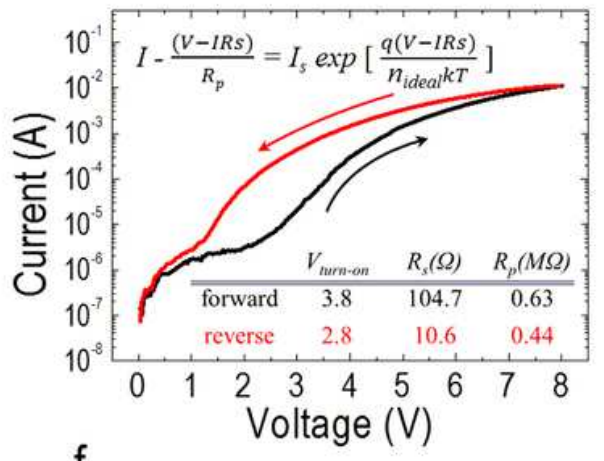

f

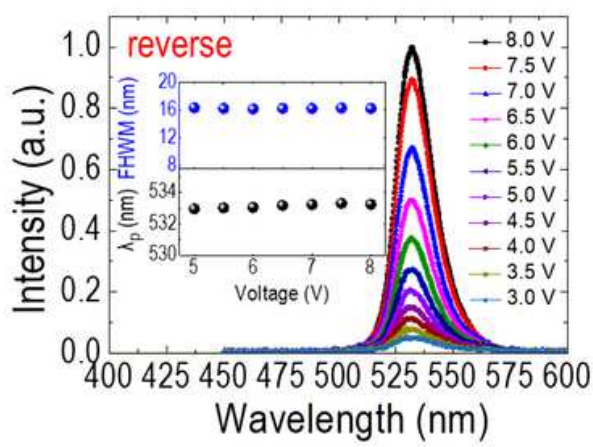

Figure 2

a Schematic illustration of ion migration inducing electric dipoles aligning along the direction of an applied electric field. Cations and anions are hence accumulated close to the Ag and ITO electrodes, respectively, leading to the formation of $\mathrm{p}$-i-n diode (bottom-up). b I-V characteristics of the perovskite LEC for the sweep voltage range of $0 \mathrm{~V} \otimes 8 \mathrm{~V} \otimes 0 \mathrm{~V}$, and a clear hysteresis effect is observed in this figure. Inset: the corresponding energy-level diagram while applying a negative poling voltage on the LEC (Ag: cathode; ITO: anode). c Re-plotting I-V characteristics of the perovskite LEC in a semi-log scale. Insets: the modified Shockley equation (top) and a summary table of the turn-on voltage, series and shunt resistances extracted from the forward and reverse I-V characteristics (bottom). $d$ EL intensity of the perovskite LEC versus sweeping voltage in a range of $0 \mathrm{~V} \otimes 8 \mathrm{~V} \otimes 0 \mathrm{~V}$. Insets show emission images of the LEC under forward (left) and reverse (right) voltages of 4, 6, and $8 \mathrm{~V}$. Evolutions of EL spectra with the increase of e forward and $f$ reverse voltages. Variation trends of the peak wavelength $(\lambda P)$ and the spectral bandwidth (FWHM) for the EL spectra with the increasing voltage are also inserted in both figures. 



Figure 3

a Illustration of resistive switching effect in the CsPbBr3 QD-based RRAM at the initial stage (left), during the migration of $\mathrm{Ag}+$ cations and $\mathrm{VBr}+$ vacancies (middle), and the final formation of the $\mathrm{VBr}$ conducting channel and the Ag filament (right). b Typical I-V characteristic of the CsPbBr3 QD-based RRAM by setting the compliance current to ICC $=1 \mathrm{~mA}$. c Re-plotted I-V characteristic of the CsPbBr3 QD-based RRAM in a log-log scale with fitted conduction mechanisms (pink and red lines represent linear Ohmic and quadratic space-charge-limited conductions in HRS, respectively, whereas green line represents linear Ohmic conduction in LRS) for SET (top) and RESET (bottom) conditions. d Retention characteristics of LRS and HRS states. Reading bias is set to $0.3 \mathrm{~V}$ in this measurement. 
a

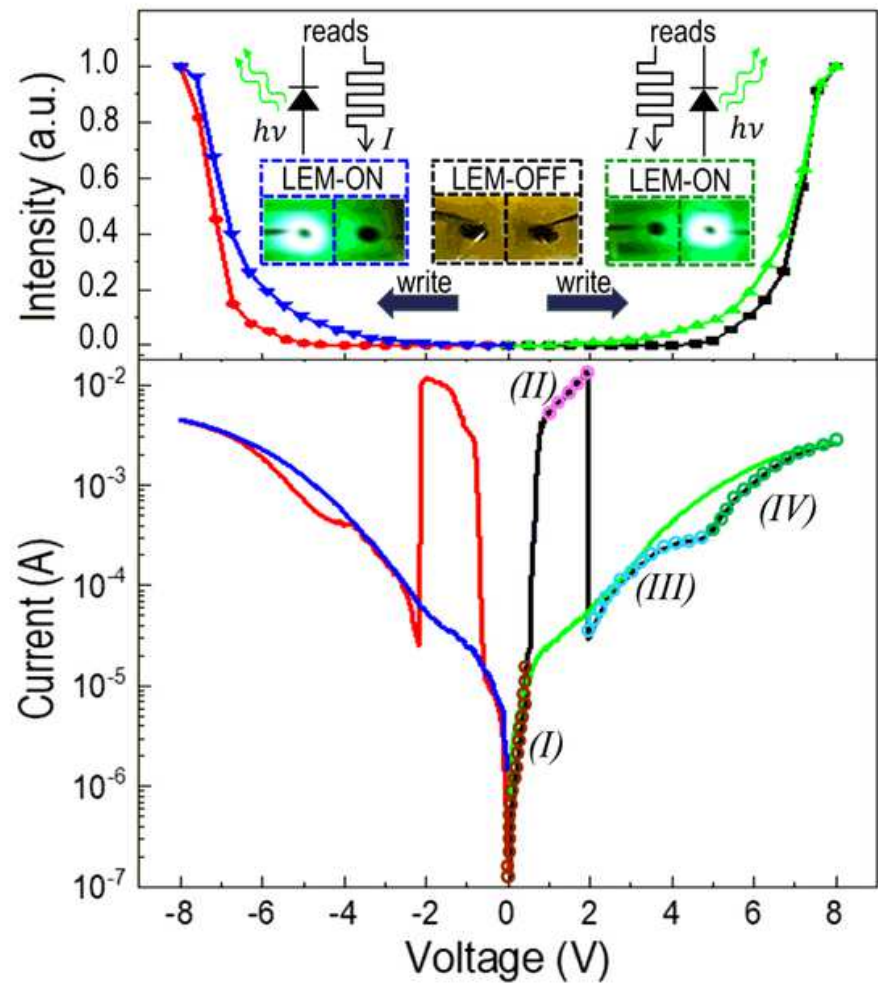

b
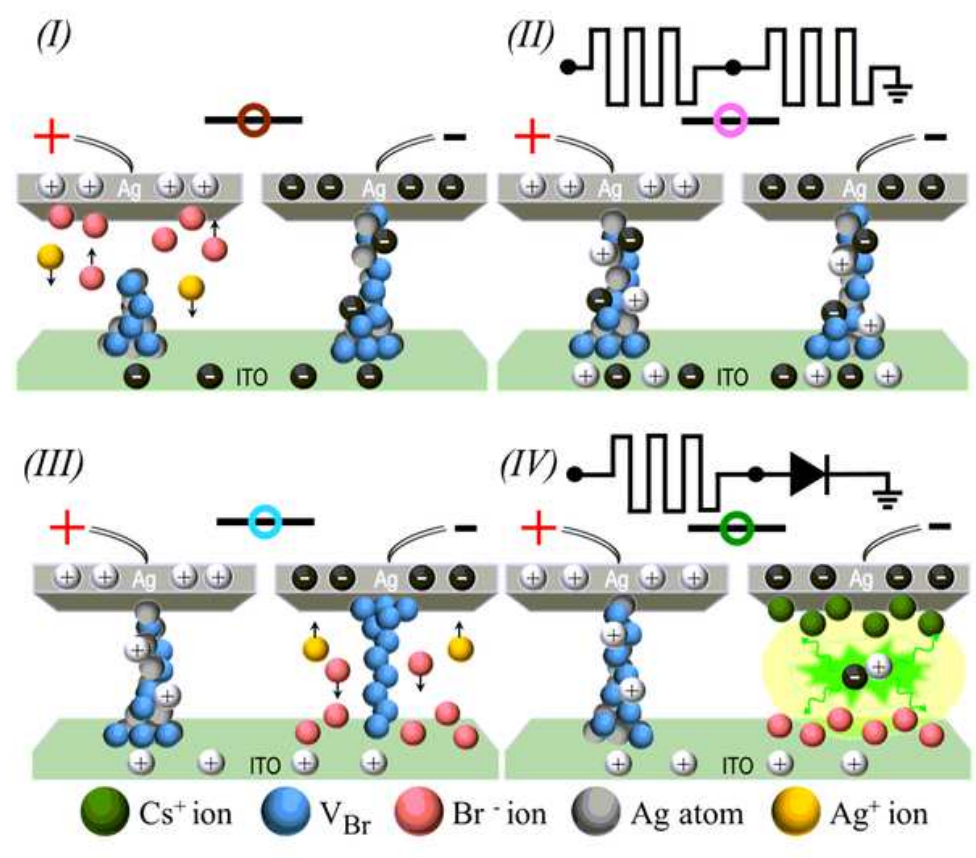

Figure 4

a Variations of EL intensity (top) and I-V characteristic (bottom) of the LEM during subjecting to a complete scanning cycle including both positive ( $0 \mathrm{~V} \Downarrow 8 \mathrm{~V} \Downarrow 0 \mathrm{~V})$ and negative $(0 \mathrm{~V} \Downarrow-8 \mathrm{~V} \Downarrow 0 \mathrm{~V})$ voltage sweeps. The positive part of the I-V characteristic is divided into four regions to clarify the underlying mechanism for the observed optoelectronic characteristics of the CsPbBr3 QD-based LEM. Insets: photographs taken while applying $0 \mathrm{~V}$ (middle), $8 \mathrm{~V}$ (right), and $-8 \mathrm{~V}$ (left) to the LEM, which addressing the LEM's potential of enabling synchronous optical and electrical readings with high modulation ability. $b$ Possible scenarios of ions migrations in accordance with individual region of (I) - (IV). 

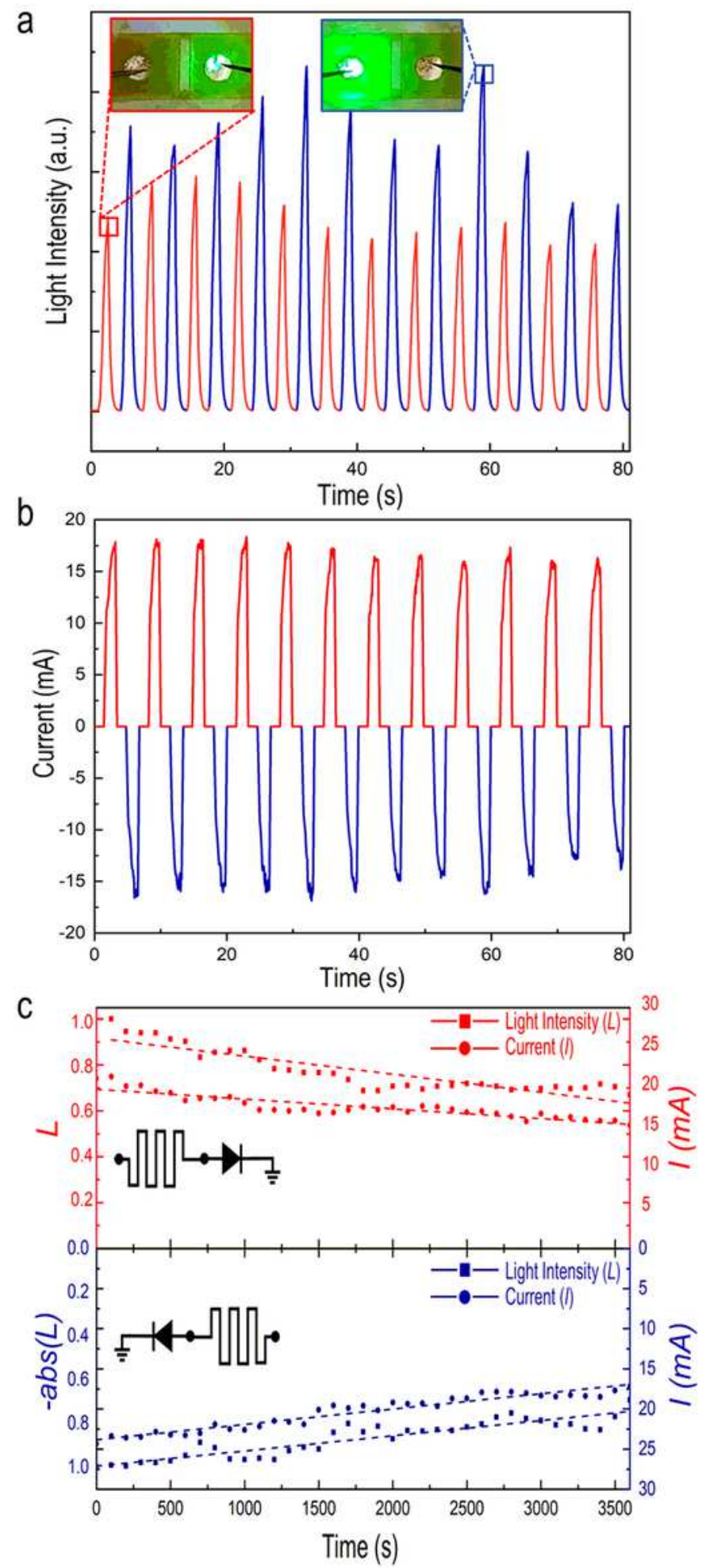

\section{Figure 5}

a Variations of LEM EL intensity versus detecting time under a modulated bias with a repetitive sequence of $8 \mathrm{~V} / 0 \mathrm{~V} /-8 \mathrm{~V} / 0 \mathrm{~V}$. Insets: photographs of emission images under different bias voltages of $8 \mathrm{~V}$ (left) and $-8 \mathrm{~V}$ (right) applied to the LEM. $\mathrm{b}$ Corresponding variation of the LEM electric current versus detecting time under the same modulated bias condition as a. c Retention characteristics by continuously reading out the EL intensity $(\mathrm{L})$ and electric current $(\mathrm{I})$ under applying bias voltages of $8 \mathrm{~V}$ (top) and $-8 \mathrm{~V}$ 
(bottom) to the LEM. The effective electrical circuits of the LEM are also plotted for different bias circumstances.

\section{Supplementary Files}

This is a list of supplementary files associated with this preprint. Click to download.

- SupportingInformationYJLee.docx 